\title{
Some Bivariate Smooth Compactly Supported Tight Framelets with Three Generators
}

\author{
A. San Antolín ${ }^{1,2}$ and R. A. Zalik \\ ${ }^{1}$ Departamento de Matemáticas, Universidad Autónoma de Madrid, 28049 Madrid, Spain \\ ${ }^{2}$ Departamento de Análisis Matemático, Universidad de Alicante, 03080 Alicante, Spain \\ ${ }^{3}$ Department of Mathematics and Statistics, Auburn University, Auburn, AL 36849-5310, USA
}

Correspondence should be addressed to A. San Antolín; angel.sanantolin@ua.es

Received 17 December 2012; Accepted 11 April 2013

Academic Editor: Sung Guen Kim

Copyright (C) 2013 A. San Antolín and R. A. Zalik. This is an open access article distributed under the Creative Commons Attribution License, which permits unrestricted use, distribution, and reproduction in any medium, provided the original work is properly cited.

\begin{abstract}
For any $2 \times 2$ dilation matrix with integer entries and $|\operatorname{det} A|=2$, we construct a family of smooth compactly supported tight wavelet frames with three generators in $L^{2}\left(\mathbb{R}^{2}\right)$. Our construction involves some compactly supported refinable functions, the oblique extension principle, and a slight generalization of a theorem of Lai and Stöckler. Estimates for the degrees of smoothness are given. With the exception of a polynomial whose coefficients must in general be computed by spectral factorization, the framelets are expressed in closed form in the frequency domain, in terms of elementary transcendental functions. By means of two examples we also show that for low degrees of smoothness the use of spectral factorization may be avoided.
\end{abstract}

\section{Introduction}

Given a $2 \times 2$ dilation matrix $A$ with integer entries, such that $|\operatorname{det} A|=2$, we construct smooth compactly supported tight framelets with three generators in $L^{2}\left(\mathbb{R}^{2}\right)$ associated to such a dilation, and with any desired degree of smoothness. Tight wavelet frames have recently become the focus of increased interest because they can be computed and applied just as easily as orthonormal wavelets, but are easier to construct.

We begin with notation and definitions. The sets of strictly positive integers, integers, and real numbers will be denoted by $\mathbb{N}, \mathbb{Z}$, and $\mathbb{R}$, respectively. Given a (Lebesgue) measurable set $S \subset \mathbb{R}^{d},|S|$ will denote its Lebesgue measure on $\mathbb{R}^{d}$ and $\chi_{S}$ will be its characteristic function. Given a matrix $M$, its transpose will be denoted by $M^{T}$ and the conjugate of its transpose will be denoted by $M^{*}$. The $n \times n$ identity matrix will be denoted by $\mathbf{I}_{n \times n}$.

We say that $A \in \mathbb{R}^{d \times d}$ is a dilation matrix preserving the lattice $\mathbb{Z}^{d}$ if all eigenvalues of $A$ have modulus greater than 1 and $A\left(\mathbb{Z}^{d}\right) \subset \mathbb{Z}^{d}$. The set of all $d \times d$ dilation matrices preserving the lattice $\mathbb{Z}^{d}$ will be denoted by $\mathbf{E}_{d}(\mathbb{Z})$. Note that if $A \in \mathbf{E}_{d}(\mathbb{Z})$, then $d_{A}:=|\operatorname{det} A|$ is an integer greater than 1.
The quotient group $\mathbb{Z}^{d} / A \mathbb{Z}^{d}$ is well defined, and by $\Delta_{A} \subset \mathbb{Z}^{d}$ we will denote a full collection of representatives of the cosets of $\mathbb{Z}^{d} / A \mathbb{Z}^{d}$. Recall that there are exactly $d_{A}$ cosets [1].

A sequence $\left\{\phi_{n}\right\}_{n=1}^{\infty}$ of elements in a separable Hilbert space $\mathbb{U}$ is a frame for $\mathbb{U}$ if there exist constants $C_{1}, C_{2}>0$ such that

$$
C_{1}\|h\|^{2} \leq \sum_{n=1}^{\infty}\left|\left\langle h, \phi_{n}\right\rangle\right|^{2} \leq C_{2}\|h\|^{2}, \quad \forall h \in \mathbb{H},
$$

where $\langle\cdot, \cdot\rangle$ denotes the inner product on $\mathbb{\uplus}$. The constants $C_{1}$ and $C_{2}$ are called frame bounds. The definition implies that a frame is a complete sequence of elements of $\mathbb{Q}$. A frame $\left\{\phi_{n}\right\}_{n=1}^{\infty}$ is tight if we may choose $C_{1}=C_{2}$.

Let $A \in \mathbf{E}_{d}(\mathbb{Z})$. A set of functions $\Psi=\left\{\psi_{1}, \ldots, \psi_{N}\right\} \subset$ $L^{2}\left(\mathbb{R}^{d}\right)$ is called a wavelet frame or framelet with dilation $A$ if the system

$$
\left\{d_{A}^{j / 2} \psi_{\ell}\left(A^{j} \mathbf{x}+\mathbf{k}\right) ; j \in \mathbb{Z}, \mathbf{k} \in \mathbb{Z}^{d}, 1 \leq \ell \leq N\right\}
$$

is a frame for $L^{2}\left(\mathbb{R}^{d}\right)$. If this system is a tight frame for $L^{2}\left(\mathbb{R}^{d}\right)$, then $\Psi$ is called a tight framelet. 
Let $\widehat{f}$ denote the Fourier transform of the function $f$. Thus, if $f \in L^{1}\left(\mathbb{R}^{d}\right), \mathbf{x}, \mathbf{t} \in \mathbb{R}^{d}$,

$$
\widehat{f}(\mathbf{t}):=\int_{\mathbb{R}^{d}} f(\mathbf{x}) e^{-2 \pi i \mathbf{x} \cdot \mathbf{t}} d \mathbf{x},
$$

where $\mathbf{x} \cdot \mathbf{t}$ denotes the dot product of vectors $\mathbf{x}$ and $\mathbf{t}$. The Fourier transform is extended to $L^{2}\left(\mathbb{R}^{d}\right)$ in the usual way.

Han [2], and independently Ron and Shen [3], found necessary and sufficient conditions for translates and dilates of a set of functions to be a tight framelet. Ron and Shen also formulated what is known as the Unitary Extension Principle (UEP), which, in addition to its other applications, provides a method for constructing compactly supported framelets. In [4] (see also [5]), Ron and Shen show two bivariate constructions of compactly supported tight framelets in $L^{2}\left(\mathbb{R}^{2}\right)$ with dilation matrix

$$
B:=\left(\begin{array}{cc}
1 & 1 \\
1 & -1
\end{array}\right)
$$

and state that constructions of compactly supported tight framelets with dilation matrix $B$ can be made in an analogous way. They also describe an algorithm for constructing compactly supported tight affine frames in $L^{2}\left(\mathbb{R}^{d}\right)$ with any dilation matrix. That algorithm works particularly well with box splines. Using Ron and Shen's method, Gröchenig and Ron [6] show how to construct, for any dilation matrix, compactly supported framelets with any desired degree of smoothness. Furthermore, based on works by Ron and Shen and by Gröchenig and Ron, Han [7] also constructs compactly supported tight wavelet frames with degree of smoothness and vanishing moments of order as large as desired. Another method for constructing smooth compactly supported tight framelets was described in [8]. Note that while in $[4,6,8]$ the number of generators of the tight framelets increases with the degree of smoothness, in [7] the number of generators may be bounded by a constant depending on the dimension and the determinant of the dilation matrix.

The remainder of this paper is organized as follows. In Section 2 we summarize results we will use for the proof of the main results. In Section 3, for any $2 \times 2$ dilation matrix $A$ with integer entries and determinant \pm 2 we describe an algorithm to construct a family of compactly supported tight framelets with three generators and with any desired degree of smoothness and dilation factor $A$. In Section 4 we illustrate the results of this paper by means of examples.

\section{Background}

In this section we summarize the results we will use in our construction of tight framelets.

The UEP led to the oblique extension principle (OEP), a method based on the UEP; it was developed by Chui et al. [9], and independently by Daubechies et al. [10], who gave the method its name. The OEP may be formulated as follows.

Theorem A. Let $A \in \mathbf{E}_{d}(\mathbb{Z})$. Let $\phi \in L^{2}\left(\mathbb{R}^{d}\right)$ be compactly supported and refinable, that is,

$$
\widehat{\phi}\left(A^{*} \mathbf{t}\right)=P(\mathbf{t}) \widehat{\phi}(\mathbf{t}),
$$

where $P(\mathbf{x})$ is a trigonometric polynomial. Assume moreover that $|\widehat{\phi}(\mathbf{0})|=1$. Let $S(\mathbf{t})$ be another trigonometric polynomial such that $S(\mathbf{t}) \geq 0$ and $S(\mathbf{0})=1$. Assume that there are trigonometric polynomials or rational functions $Q_{\ell}, \ell=$ $1, \ldots, N$, that satisfy the OEP condition

$$
\begin{aligned}
S\left(A^{*} \mathbf{t}\right) & P(\mathbf{t}) \overline{P(\mathbf{t}+\mathbf{j})}+\sum_{\ell=1}^{N} Q_{\ell}(\mathbf{t}) \overline{Q_{\ell}(\mathbf{t}+\mathbf{j})} \\
\quad & \begin{cases}S(\mathbf{t}) & \text { if } \mathbf{j} \in \mathbb{Z}^{d}, \\
0 & \text { if } \mathbf{j} \in\left(\frac{\left(A^{*}\right)^{-1}\left(\mathbb{Z}^{d}\right)}{\mathbb{Z}^{d}}\right) \backslash \mathbb{Z}^{d} .\end{cases}
\end{aligned}
$$

If

$$
\widehat{\psi_{\ell}}\left(A^{*} \mathbf{t}\right):=Q_{\ell}(\mathbf{t}) \widehat{\phi}(\mathbf{t}), \quad \ell=1, \ldots, N,
$$

then $\Psi=\left\{\psi_{1}, \ldots, \psi_{N}\right\}$ is a tight framelet in $L^{2}\left(\mathbb{R}^{d}\right)$ with dilation factor $A$ and frame constant 1 .

With an additional decay condition, Theorem A follows from [10, Proposition 1.11], except for the value of the frame constant, which follows from, for example, [3, Theorem 6.5]. However, recent results of Han imply that this decay condition is redundant. Indeed, Theorem A in its present formulation is a consequence of Proposition 4, Corollary 12, and Theorem 17 in [11] (for a simpler version of Han's results in dimension, see [12]).

We also need the following slight generalization of Theorem 3.4 of Lai and Stöckler [13]. The proof is similar and will be omitted. We have also included in the statement a generalization of the algorithm implicit in the proof of Theorem 3.4.

Theorem B. Let $A \in \mathbf{E}_{d}(\mathbb{Z})$ and let $\Delta_{A}=\left\{\mathbf{q}_{s}\right\}_{s=0}^{d_{A}-1}$ and $\Delta_{A^{*}}=\left\{\mathbf{p}_{s}\right\}_{s=0}^{d_{A}-1}$ be full collections of representatives of the cosets of $\mathbb{Z}^{d} / A \mathbb{Z}^{d}$ and $\mathbb{Z}^{d} / A^{*} \mathbb{Z}^{d}$, respectively, with $\mathbf{q}_{0}=\mathbf{p}_{0}=\mathbf{0}$. Let $P(\mathbf{t})$ be a trigonometric polynomial defined on $\mathbb{R}^{d}$ that satisfies the condition:

$$
\sum_{s=0}^{d_{A}-1}\left|P\left(\mathbf{t}+\left(A^{*}\right)^{-1}\left(\mathbf{p}_{s}\right)\right)\right|^{2} \leq 1,
$$

let

$$
\mathscr{P}(\mathbf{t}):=\left(P\left(\mathbf{t}+\left(A^{*}\right)^{-1}\left(\mathbf{p}_{s}\right)\right) ; s=0, \ldots, d_{A}-1\right)^{T},
$$

and let

$$
\mathscr{M}(\mathbf{t}):=d_{A}^{-1 / 2}\left(e^{i 2 \pi \mathbf{q} l \cdot \mathbf{t}+\left(\left(A^{*}\right)^{-1}\left(\mathbf{p}_{s}\right)\right)} ; \ell, s=0, \ldots, d_{A}-1\right)
$$

be the polyphase matrix, where $s$ denotes the row index and $l$ denotes the column index.

Let the $d_{A} \times 1$ matrix function $G(\mathbf{t})$ be defined by

$$
G(\mathbf{t}):=\mathscr{M}^{*}(\mathbf{t}) \mathscr{P}(\mathbf{t})=\left(L_{k}\left(A^{*} \mathbf{t}\right) ; k=0, \ldots, d_{A}-1\right)^{T},
$$


and suppose that there exist trigonometric polynomials $\widetilde{P}_{1}$, $\ldots, \widetilde{P}_{M}$ such that

$$
\sum_{k=0}^{d_{A}-1}\left|L_{k}(\mathbf{t})\right|^{2}+\sum_{j=1}^{M}\left|\widetilde{P}_{j}(\mathbf{t})\right|^{2}=1
$$

Let $N:=d_{A}+M$ and let the $N \times 1$ matrix function $\mathscr{G}(\mathbf{t})$ be defined by

$$
\begin{gathered}
\mathscr{G}(\mathbf{t}):=\left(L_{k}\left(A^{*} \mathbf{t}\right) ; k=0, \ldots, d_{A}-1,\right. \\
\left.\widetilde{P}_{j}\left(A^{*} \mathbf{t}\right) ; 1 \leq j \leq M\right)^{T}, \\
\widetilde{Q}(\mathbf{t}):=I_{N \times N}-\mathscr{G}(\mathbf{t}) \mathscr{G}^{*}(\mathbf{t}) .
\end{gathered}
$$

Let $K(\mathbf{t})$ denote the first $d_{A} \times N$ block matrix of $\widetilde{Q(\mathbf{t})}$,

$$
Q(\mathbf{t}):=\mathscr{M}(\mathbf{t}) K(\mathbf{t}),
$$

and let $\left[Q_{1}(\mathbf{t}), \ldots, Q_{N}(\mathbf{t})\right]$ denote the first row of $Q(\mathbf{t})$. Then the trigonometric polynomials $P$ and $Q_{\ell}, \ell=1, \ldots, N$, satisfy the identity (6) with $S(\mathbf{t})=1$.

Using the OEP and Theorem B, we will obtain a general method for constructing compactly supported framelets in $L^{2}\left(\mathbb{R}^{2}\right)$ valid for any dilation matrix.

We will use the following theorem of Gröchenig and Madych [1].

Theorem C. Let $A \in \mathbf{E}_{d}(\mathbb{Z})$ and let $\Delta_{A}=\left\{\mathbf{q}_{s}\right\}_{s=0}^{d_{A}-1}$ be a full collection of representatives of the cosets of $\mathbb{Z}^{d} / A \mathbb{Z}^{d}$ with $\mathbf{q}_{0}=$ $\mathbf{0}$. Then the characteristic function $\chi_{E}$, where the set $E$ is defined by

$$
E:=\left\{\mathbf{x} \in \mathbb{R}^{d}: \mathbf{x}=\sum_{j=1}^{\infty} A^{-j} \mathbf{k}^{(j)}, \mathbf{k}^{(j)} \in \Delta_{A}\right\}
$$

is a nonnull compactly supported measurable function, $\left\|\chi_{E}\right\|_{L^{2}\left(\mathbb{R}^{d}\right)} \geq 1$, and it satisfies the refinement equation:

$$
\begin{aligned}
& \widehat{\chi_{E}}\left(A^{*}(\mathbf{t})\right)=H(\mathbf{t}) \widehat{\chi_{E}}(\mathbf{t}), \text { where } \\
& H(\mathbf{t}):=\frac{1}{d_{A}} \sum_{s=0}^{d_{A}-1} e^{-2 \pi i \mathbf{t} \cdot \mathbf{q}_{s}} .
\end{aligned}
$$

The following statement may be found in [14, Appendix A.2]. The proof is straightforward and will be omitted.

Lemma D. Let $C^{0}$ be the class of continuous functions in $L^{2}\left(\mathbb{R}^{d}\right)$, and let $C^{r}, r=1,2, \ldots$ be the class of functions $f$ such that all partial derivatives of $f$ of order not greater than $r$ are continuous and in $L^{2}\left(\mathbb{R}^{d}\right)$. If

$$
|\widehat{f}(\mathbf{t})| \leq C(1+|\mathbf{t}|)^{-N-\varepsilon}
$$

for some integer $N \geq d$ and $\varepsilon>0$, then $f$ is in $C^{N-d}$.
Proofs of the following proposition may be found in $[15$, Lemma 3.1], [14, Proposition 5.23], or [6, Result 2.6].

Proposition E. Let $A \in \mathbf{E}_{d}(\mathbb{Z})$ and let $\Delta_{A}=\left\{\mathbf{q}_{s}\right\}_{s=0}^{d_{A}-1}$ be a full collection of representatives of the cosets of $\mathbb{Z}^{d} / A \mathbb{Z}^{d}$ with $\mathbf{q}_{0}=\mathbf{0}$, and let $E \subset \mathbb{R}^{d}$ be the set defined by (15). Then there exist two positive constants $\varepsilon$ and $C$ such that

$$
\left|\widehat{\chi_{E}}(\mathbf{t})\right| \leq C|\mathbf{t}|^{-\varepsilon} \text {. }
$$

\section{A Family of Tight Framelets}

Let $A \in \mathbb{Z}^{2 \times 2}$ be a dilation matrix with integer entries such that $|\operatorname{det} A|=2$. In this section we construct smooth compactly supported tight framelets with three generators in $L^{2}\left(\mathbb{R}^{2}\right)$ with dilation factor $A$ and any desired degree of smoothness.

Two matrices $A$ and $B$ with integer coefficients are integrally similar if there exists a matrix $U$ with integer entries such that $|\operatorname{det} U|=1$ and $A=U^{-1} B U$. Let

$$
\begin{array}{rlr}
A_{1}:=\left(\begin{array}{cc}
0 & 2 \\
1 & 0
\end{array}\right), & A_{2}:=\left(\begin{array}{cc}
0 & 2 \\
-1 & 0
\end{array}\right), \\
A_{3}:=\left(\begin{array}{cc}
0 & 2 \\
-1 & 1
\end{array}\right), & A_{4}:=\left(\begin{array}{cc}
0 & -2 \\
1 & -1
\end{array}\right), \\
A_{5}:=\left(\begin{array}{cc}
1 & 1 \\
-1 & 1
\end{array}\right), & A_{6}:=\left(\begin{array}{cc}
-1 & -1 \\
1 & -1
\end{array}\right) .
\end{array}
$$

The following complete classification of all matrices in $\mathbf{E}_{2}(\mathbb{Z})$ with $|\operatorname{det} A|=2$ was found by Lagarias and Wang [16, Lemma $5.2]$.

Lemma F. Let $A \in \mathbf{E}_{2}(\mathbb{Z})$. If $\operatorname{det} A=-2$, then $A$ is integrally similar to $A_{1}$. If $\operatorname{det} A=2$, then $A$ is integrally similar to one of the matrices $A_{k}, k=2, \ldots, 6$.

We now focus on the dilation matrices $A_{k}$. For $r \in N$, let

$$
\begin{gathered}
\Lambda^{(r)}:=\left\{\mathbf{q}_{0}=(0,0)^{T}, \mathbf{q}_{1}=(2 r-1,0)^{T}\right\}, \\
\Gamma:=\left\{\mathbf{p}_{0}=(0,0)^{T}, \mathbf{p}_{1}=(0,1)^{T}\right\} .
\end{gathered}
$$

It is easy to see that for each $r \in \mathbb{N}$ the set $\Lambda^{(r)}$ is a full collection of representatives of the cosets of $\mathbb{Z}^{2} / A_{k} \mathbb{Z}^{2}, k=$ $1, \ldots, 6$, and that $\Gamma$ is a full collection of representatives of the cosets of $\mathbb{Z}^{2} / A_{k}^{*} \mathbb{Z}^{2}, k=1, \ldots, 6$. Moreover, we have

$$
\begin{gathered}
\left\{\left(A_{k}^{*}\right)^{-1} \mathbf{p}_{0},\left(A_{k}^{*}\right)^{-1} \mathbf{p}_{1}\right\}=\left\{(0,0)^{T},\left(\frac{1}{2}, 0\right)^{T}\right\}, \quad k=1,2,3, \\
\left\{\left(A_{4}^{*}\right)^{-1} \mathbf{p}_{0},\left(A_{4}^{*}\right)^{-1} \mathbf{p}_{1}\right\}=\left\{\left(0,\left(-\frac{1}{2}, 0\right)\right)^{T}\right\}, \\
\left\{\left(A_{5}^{*}\right)^{-1} \mathbf{p}_{0},\left(A_{5}^{*}\right)^{-1} \mathbf{p}_{1}\right\}=\left\{(0,0)^{T},\left(\frac{1}{2}, \frac{1}{2}\right)^{T}\right\}, \\
\left\{\left(A_{6}^{*}\right)^{-1} \mathbf{p}_{0},\left(A_{6}^{*}\right)^{-1} \mathbf{p}_{1}\right\}=\left\{(0,0)^{T},\left(-\frac{1}{2},-\frac{1}{2}\right)^{T}\right\} .
\end{gathered}
$$


For simplicity we shall consider $\Lambda^{(1)}$ as in (20) with $r=1$, and $\Gamma$ as in (21). Then the corresponding matrix $\mathscr{M}(\mathbf{t}), \mathbf{t}=$ $\left(t_{1}, t_{2}\right)$, defined by (10), where $A$ is any of the matrices $A_{k}$, $k=1, \ldots, 6$, is

$$
\mathscr{M}(\mathbf{t})=\frac{1}{\sqrt{2}}\left(\begin{array}{cc}
1 & e^{i 2 \pi t_{1}} \\
1 & -e^{i 2 \pi t_{1}}
\end{array}\right) .
$$

We now construct a family of smooth compactly supported refinable functions in $L^{2}\left(\mathbb{R}^{2}\right)$ with dilation factor $A_{k}$.

Proposition 1. Let the matrices $A_{k}, k=1, \ldots, 6$ be defined as in (19). Let $m, n \in \mathbb{N}, \mathbf{t}=\left(t_{1}, t_{2}\right)$ and $P(\mathbf{t}):=\cos ^{2 n}(2 m-1) \pi t_{1}$. Let

$$
\widehat{\phi}(\mathbf{t}):=\left.\left.|| E\right|^{-1} \widehat{\chi}_{E}(\mathbf{t})\right|^{2 n}
$$

where $E$ is the set defined by (15) with $\Delta_{A}=\Lambda^{(m)}$, and $A$ is one of the matrices $A_{k}$. Then $\widehat{\phi}(\mathbf{0})=1$, and the function $\phi$ whose Fourier transform $\widehat{\phi}$ is defined by (24) has the following properties: $\phi$ is nonnull, compactly supported, and square integrable on $\mathbb{R}^{2},\|\phi\|_{L^{2}\left(\mathbb{R}^{2}\right)} \leq 1$, and satisfies the refinement equation:

$$
\widehat{\phi}\left(A_{k}^{*}(\mathbf{t})\right)=P(\mathbf{t}) \widehat{\phi}(\mathbf{t}) .
$$

Moreover, if $2 \varepsilon n-2>r>1$, where $\varepsilon$ is defined in Proposition $E$, then $\phi$ is in continuity class $C^{r}$.

Proof. From Theorem $\mathrm{C}$ we deduce that $\chi_{E}$ is a nonnull compactly supported function.

We now prove that $\|\phi\|_{L^{2}\left(\mathbb{R}^{2}\right)} \leq 1$. Let $\varphi_{1}:=|E|^{-1} \chi_{E}$ and for $j \in \mathbb{N}$ let $\varphi_{j}$ denote the $j$-fold convolution of $\varphi_{1}$ with itself. Since $\|\phi\|_{L^{2}\left(\mathbb{R}^{2}\right)}=\|\phi\|_{L^{2}\left(\mathbb{R}^{2}\right)}$, by Young's inequality for convolutions and bearing in mind that $|E| \geq 1$, we conclude that

$$
\begin{aligned}
\|\phi\|_{L^{2}\left(\mathbb{R}^{d}\right)} & \leq\left\|\varphi_{n-1}\right\|_{L^{2}\left(\mathbb{R}^{d}\right)}\left\||E|^{-1} \chi_{E}\right\|_{L^{1}\left(\mathbb{R}^{d}\right)} \\
& =\left\|\varphi_{n-1}\right\|_{L^{2}\left(\mathbb{R}^{d}\right)} \leq \cdots \leq\left\|\varphi_{1}\right\|_{L^{2}\left(\mathbb{R}^{d}\right)} \leq 1 .
\end{aligned}
$$

We now show that $\widehat{\phi}$ satisfies the refinement equation (25). From (16) we have

$$
\begin{aligned}
\widehat{\phi}\left(A_{k}^{*}(\mathbf{t})\right) & =|E|^{-2 n}\left|\widehat{\chi_{E}}\left(A_{k}^{*}(\mathbf{t})\right)\right|^{2 n} \\
& =|H(\mathbf{t})|^{2 n}|E|^{-2 n}\left|\widehat{\chi_{E}}(\mathbf{t})\right|^{2 n}=P(\mathbf{t}) \widehat{\phi}(\mathbf{t}),
\end{aligned}
$$

where $H(\mathbf{t})=(1 / 2)\left(1+e^{2 \pi i(2 m-1) t_{1}}\right)$. 1.

Since $\widehat{\chi}_{E}(\mathbf{0})=|E|$, the definition of $\widehat{\phi}$ implies that $\widehat{\phi}(\mathbf{0})=$

We now prove the estimates for the degree of smoothness of $\phi$. By Proposition E, we have

$$
\begin{aligned}
|\widehat{\phi}(\mathbf{t})| & =|E|^{-2 n}\left|\widehat{\chi_{E}}(\mathbf{t})\right|^{2 n} \\
& \leq C|E|^{-2 n}|\mathbf{t}|^{-2 \varepsilon n} \leq C|\mathbf{t}|^{-2 \varepsilon n} .
\end{aligned}
$$

Moreover, since $\widehat{\phi}$ is continuous,

$$
|\widehat{\phi}(\mathbf{t})| \leq D(1+|\mathbf{t}|)^{-2 \varepsilon n} .
$$

Hence, if $2 \varepsilon n-2>r>1$, Lemma D implies that $\phi$ is in continuity class $C^{r}$.

Finally, replicating an argument of Wojtaszczyk [14, page $79]$ it is easy to see that $\phi$ is a compactly supported function on $\mathbb{R}^{2}$.

We now construct tight framelets $\Psi=\left\{\psi_{0}, \psi_{1}, \psi_{2}\right\}$ in $L^{2}\left(\mathbb{R}^{2}\right)$ with dilation matrices $A \in \mathbf{E}_{2}(\mathbb{Z})$ such that the functions $\psi_{\rho}$ are smooth and compactly supported. For this purpose we use the refinable functions that we obtained in Proposition 1, Theorem B, the Oblique Extension Principle, and an appropriate change of variables.

Let $A_{k}, k=1, \ldots, 6$ be the matrices defined in (19), let $\Gamma$ be defined by (21), assume that $n, m \in \mathbb{N}, \mathbf{t}=\left(t_{1}, t_{2}\right) \in \mathbb{R}^{2}$, and that $P(\mathbf{t})$ defined as in Proposition 1. Using (22) we readily see that

$$
\begin{aligned}
& |P(\mathbf{t})|^{2}+\left|P\left(\mathbf{t}+\left(A_{k}^{*}\right)^{-1} \mathbf{p}_{1}\right)\right|^{2} \\
& =\cos ^{4 n}\left((2 m-1) \pi t_{1}\right) \\
& \quad+\cos ^{4 n}\left((2 m-1) \pi\left(t_{1}+\frac{1}{2}\right)\right),
\end{aligned}
$$

and by an elementary computation we conclude that

$$
|P(\mathbf{t})|^{2}+\left|P\left(\mathbf{t}+\left(A_{k}^{*}\right)^{-1} \mathbf{p}_{1}\right)\right|^{2} \leq 1,
$$

and that equality holds only if $2(2 m-1) t_{1} \in \mathbb{Z}$. Since the values of the trigonometric polynomial $P$ in Proposition 1 only depend on one variable, from a lemma of Riesz (cf., e.g., [17, Lemma 6.1.3], [14, Lemma 4.6]) we know there is a nonnull trigonometric polynomial $L\left(A_{k}^{*} \mathbf{t}\right)$ on $\mathbb{R}^{2}$ such that

$$
\begin{aligned}
\left|L\left(A_{k}^{*} \mathbf{t}\right)\right|^{2} & =1-\left(|P(\mathbf{t})|^{2}+\left|P\left(\mathbf{t}+\left(A_{k}^{*}\right)^{-1} \mathbf{p}_{1}\right)\right|^{2}\right) \\
& =1-\cos ^{4 n}\left((2 m-1) \pi t_{1}\right)-\sin ^{4 n}\left((2 m-1) \pi t_{1}\right) .
\end{aligned}
$$

The coefficients of $L(\mathbf{t})$ may be obtained by spectral factorization ([18]).

The following theorem describes the construction of smooth tight framelets of compact support and arbitrary degree of smoothness with dilation matrix $A_{k}$.

Theorem 2. Let $A_{k}, k=1, \ldots, 6$. Let $m, n \in \mathbb{N}, \mathbf{t}=\left(t_{1}, t_{2}\right)$, let $P(\mathbf{t}):=\cos ^{2 n}\left((2 m-1) \pi t_{1}\right)$, and let

$$
\begin{aligned}
Q_{1}(\mathbf{t}):=\frac{1}{\sqrt{2}}[ & 1-\cos ^{4 n}\left((2 m-1) \pi t_{1}\right) \\
& -\cos ^{2 n}\left((2 m-1) \pi t_{1}\right) \\
& \left.\times \sin ^{2 n}\left((2 m-1) \pi t_{1}\right)\right],
\end{aligned}
$$




$$
\begin{aligned}
& Q_{2}(\mathbf{t}):=\frac{e^{i 2 \pi t_{1}}}{\sqrt{2}}\left[1-\cos ^{4 n}\left((2 m-1) \pi t_{1}\right)\right. \\
& +\cos ^{2 n}\left((2 m-1) \pi t_{1}\right) \\
& \left.\times \sin ^{2 n}\left((2 m-1) \pi t_{1}\right)\right], \\
& Q_{3}(\mathbf{t}):=-\frac{1}{2} \cos ^{2 n}\left((2 m-1) \pi t_{1}\right) \overline{L\left(A_{k}^{*} \mathbf{t}\right)},
\end{aligned}
$$

where $L\left(A_{k}^{*} \mathbf{t}\right)$ is a trigonometric polynomial that satisfies (32). If $\widehat{\phi}$ is defined by (24),

$$
\widehat{\psi_{\ell}}\left(A_{k}^{*} \mathbf{t}\right):=Q_{\ell}(\mathbf{t}) \widehat{\phi}(\mathbf{t}), \quad \ell=1,2,3
$$

and $\Psi=\left\{\psi_{1}, \psi_{2}, \psi_{3}\right\}$ is the set of inverse Fourier transforms of the functions $\widehat{\psi}_{\ell}$ defined in the preceding displayed identity, then $\Psi$ is a tight framelet with dilation factor $A_{k}$ and frame constant 1 , and the functions $\psi_{\ell}$ have compact support. Moreover, if $2 \varepsilon n-2>r>1$, where $\varepsilon$ is defined in Proposition $E$, then the functions $\psi_{\ell}(\mathbf{t})$ are in continuity class $C^{r}$.

Proof. Recall that the set $\Lambda^{(m)}$ defined by (20) is a full collections of representatives of the cosets of $\mathbb{Z}^{2} / A_{k} \mathbb{Z}^{2}$, and that the set $\Gamma$ defined by (21) is a full collection of representatives of the cosets of $\mathbb{Z}^{2} / A_{k}^{*} \mathbb{Z}^{2}$. Thus, the inequality (31) allows us to apply Theorem $\mathrm{B}$.

From (9) we see that

$$
\mathscr{P}(\mathbf{t})=\left(\cos ^{2 n}\left((2 m-1) \pi t_{1}\right), \sin ^{2 n}\left((2 m-1) \pi t_{1}\right)\right)^{T},
$$

whence (23) implies that

$$
\begin{gathered}
L_{0}\left(A_{k}^{*} \mathbf{t}\right)=\frac{1}{\sqrt{2}}\left(\cos ^{2 n}\left((2 m-1) \pi t_{1}\right)+\sin ^{2 n}\left((2 m-1) \pi t_{1}\right)\right), \\
L_{1}\left(A_{k}^{*} \mathbf{t}\right)=\frac{1}{\sqrt{2}} e^{-i 2 \pi t_{1}}\left(\cos ^{2 n}\left((2 m-1) \pi t_{1}\right)\right. \\
\left.-\sin ^{2 n}\left((2 m-1) \pi t_{1}\right)\right) .
\end{gathered}
$$

Thus, we have

$$
\begin{aligned}
\left|L_{0}\left(A_{k}^{*} \mathbf{t}\right)\right|^{2}+\left|L_{1}\left(A_{k}^{*} \mathbf{t}\right)\right|^{2}= & \cos ^{4 n}\left((2 m-1) \pi t_{1}\right) \\
& +\sin ^{4 n}\left((2 m-1) \pi t_{1}\right) .
\end{aligned}
$$

Let $L$ be defined as in (32). Setting $\widetilde{P}_{1}:=L$ we therefore conclude that

$$
\left|L_{0}(\mathbf{t})\right|^{2}+\left|L_{1}(\mathbf{t})\right|^{2}+\left|\widetilde{P}_{1}(\mathbf{t})\right|^{2}=1 \text {. }
$$

Applying the algorithm described in Theorem B with $N=3$ and $\widetilde{P}_{1}=L$, and bearing in mind that $\overline{L_{0}}=L_{0}$, we obtain

$$
\begin{aligned}
& Q_{1}(\mathbf{t})=\frac{1}{\sqrt{2}}\left[1-\left|L_{0}\left(A_{k}^{*} \mathbf{t}\right)\right|^{2}\right.\left.-e^{i 2 \pi(2 b-1) t_{1}} L_{1}\left(A_{k}^{*} \mathbf{t}\right) L_{0}\left(A_{k}^{*} \mathbf{t}\right)\right] \\
&=\frac{1}{\sqrt{2}}\left[1-\cos ^{4 n}\left((2 m-1) \pi t_{1}\right)\right.\left.\quad-\cos ^{2 n}\left((2 m-1) \pi t_{1}\right) \sin ^{2 n}\left((2 m-1) \pi t_{1}\right)\right], \\
& Q_{2}(\mathbf{t})= \frac{1}{\sqrt{2}}\left[e^{i 2 \pi t_{1}}-L_{0}\left(A_{k}^{*} \mathbf{t}\right) \overline{L_{1}\left(A_{k}^{*} \mathbf{t}\right)}\right. \\
&\left.-e^{i 2 \pi t_{1}}\left|L_{1}\left(A_{k}^{*} \mathbf{t}\right)\right|^{2}\right] \\
&= \frac{e^{i 2 \pi t_{1}}}{\sqrt{2}}\left[1-\cos ^{4 n}\left((2 m-1) \pi t_{1}\right)\right. \\
& \quad+\cos ^{2 n}\left((2 m-1) \pi t_{1}\right) \\
&\left.\quad \times \sin ^{2 n}\left((2 m-1) \pi t_{1}\right)\right], \\
& Q_{3}(\mathbf{t})=-\frac{1}{\sqrt{2}}\left[L_{0}\left(A_{k}^{*} \mathbf{t}\right)+e^{i 2 \pi t_{1}} L_{1}\left(A_{k}^{*} \mathbf{t}\right)\right] \overline{L\left(A_{k}^{*} \mathbf{t}\right)} \\
& \cos ^{2 n}\left((2 m-1) \pi t_{1}\right) \overline{L\left(A_{k}^{*} \mathbf{t}\right) .} \\
&=
\end{aligned}
$$

Theorem B guarantees that the trigonometric polynomials $P, Q_{0}, Q_{1}$ and $Q_{2}$, satisfy the identity (6) with $S(x)=1$ and $N=3$. This may also be verified by direct computation. Applying Theorem A we conclude that $\Psi$ is a tight framelet in $L^{2}\left(\mathbb{R}^{2}\right)$.

Since the functions $Q_{\ell}$ are trigonometric polynomials and therefore bounded on $\mathbb{R}^{2}$, the smoothness of the functions $\psi_{\ell}$ follows from (29) and Lemma D.

Finally, note that the functions $\psi_{\ell}$ are compactly supported because $\phi$ is compactly supported and the $Q_{\ell}$ are trigonometric polynomials.

Using Theorem 2 and Lemma F we now obtain an algorithm for constructing tight framelets in $L^{2}\left(\mathbb{R}^{2}\right)$ with dilation factor $A$, for any matrix $A \in \mathbb{R}^{2 \times 2}$ preserving the lattice $\mathbb{Z}^{2}$ and with $|\operatorname{det} A|=2$. These framelets have three compactly supported generators of arbitrary degree of smoothness.

Corollary 3. Let $A \in \mathbf{E}_{2}(\mathbb{Z})$ with $|\operatorname{det} A|=2$ and let $k \in$ $\{1, \ldots, 6\}$ be such that there exists a matrix $U \in \mathbb{Z}^{2 \times 2}$ with $\operatorname{det}(U)=1$, such that $A=U^{-1} A_{k} U$. Let $m, n \in \mathbb{N}, \mathbf{t}=\left(t_{1}, t_{2}\right)$, $P(\mathbf{t}):=\cos ^{2 n}(2 m-1) \pi t_{1}$, let $\widehat{\phi}$ be defined by (24), and let $\Psi=\left\{\psi_{1}, \psi_{2}, \psi_{3}\right\}$ be the set of inverse Fourier transforms of the functions $\widehat{\psi_{\ell}}$ defined by (34). If

$$
\theta_{\ell}(\mathbf{t})=\psi_{\ell}(U \mathbf{t}), \quad \ell=1,2,3,
$$


then $\Theta=\left\{\theta_{1}, \theta_{2}, \theta_{3}\right\} \subset L^{2}\left(\mathbb{R}^{2}\right)$ is a tight framelet with $A$, and the functions $\theta_{\ell}$ have compact support. Moreover, if $2 \varepsilon n-2>$ $r>1$, where $\varepsilon$ is defined in Proposition $E$, then the functions $\theta_{\ell}(\mathbf{t})$ are in continuity class $C^{r}$.

Proof. Since $U^{-1} \mathbb{Z}^{2}=\mathbb{Z}^{2}$, the assertion that $\Theta$ is a tight framelet in $L^{2}\left(\mathbb{R}^{2}\right)$ with frame constant 1 readily follows by a change of variable of the form $t \rightarrow U^{-1} t$.

Let $r$ be an integer such that $2 \varepsilon n-2>r>1$, where $\varepsilon$ is defined in Proposition E. Since $\psi_{\ell}$ is in continuity class $C^{r}$, applying the chain rule we conclude that also $\theta_{\ell}$ is in continuity class $C^{r}$.

\section{Examples}

In this section, we illustrate the results of this paper showing examples of tight framelets where the use of spectral factorization may be avoided.

Example 4. Let $A_{k}, k \in\{1, \ldots, 6\}, m \in \mathbb{N}, n=1, \mathbf{t}=\left(t_{1}, t_{2}\right)$, $P(\mathbf{t}):=\cos ^{2}\left((2 m-1) \pi t_{1}\right)$ and $\Gamma$ be the set defined by $(21)$. We can obtain a tight framelet by elementary computations. Since

$$
\begin{aligned}
1-|P(\mathbf{t})|^{2} & -\left|P\left(\mathbf{t}+\left(A_{k}^{*}\right)^{-1} \mathbf{p}_{1}\right)\right|^{2} \\
= & 1-\cos ^{4}\left((2 m-1) \pi t_{1}\right) \\
& -\sin ^{4}\left((2 m-1) \pi t_{1}\right) \\
= & 2^{-1} \sin ^{2}\left(2(2 m-1) \pi t_{1}\right),
\end{aligned}
$$

a choice for $L(t)$ is $L(t):=(1 / \sqrt{2}) \sin \left(2(2 m-1) \pi t_{1}\right)$. Thus, if we set

$$
\begin{aligned}
& Q_{1}(\mathbf{t})=\frac{1}{\sqrt{2}}\left[1-\cos ^{4}\left((2 m-1) \pi t_{1}\right)\right. \\
& \left.-\cos ^{2}\left((2 m-1) \pi t_{1}\right) \sin ^{2}\left((2 m-1) \pi t_{1}\right)\right], \\
& Q_{2}(\mathbf{t})=\frac{e^{i 2 \pi t_{1}}}{\sqrt{2}}\left[1-\cos ^{4}\left((2 m-1) \pi t_{1}\right)\right. \\
& \left.+\cos ^{2}\left((2 m-1) \pi t_{1}\right) \sin ^{2}\left((2 m-1) \pi t_{1}\right)\right], \\
& Q_{3}(\mathbf{t})=-\frac{1}{2 \sqrt{2}} \cos ^{2}\left((2 m-1) \pi t_{1}\right) \sin \left(2(2 m-1) \pi t_{1}\right) \text {, }
\end{aligned}
$$

and if $\widehat{\phi}$ is defined by (24) with $n=1$,

$$
\widehat{\psi_{\ell}}\left(A_{k}^{*} \mathbf{t}\right):=Q_{\ell}(\mathbf{t}) \widehat{\phi}(\mathbf{t}), \quad \ell=1,2,3,
$$

and $\Psi=\left\{\psi_{1}, \psi_{2}, \psi_{3}\right\}$ is the set of inverse Fourier transforms of the functions $\widehat{\psi}_{\ell}$ defined in the preceding displayed identity, then $\Psi$ is a tight framelet in $L^{2}\left(\mathbb{R}^{2}\right)$ with dilation factor $A_{k}$, and the functions $\psi_{\ell}$ are compactly supported.

Example 5. Let $A_{k}, k \in\{1, \ldots, 6\}, m \in \mathbb{N}, n=2, \mathbf{t}=\left(t_{1}, t_{2}\right)$, $P(\mathbf{t}):=\cos ^{4}\left((2 m-1) \pi t_{1}\right)$ and $\Gamma$ be the set defined by $(21)$.
The case when $n=2$ is also sufficiently simple that it does not require the use of spectral factorization algorithms to compute $L(\mathbf{t})$. We will just apply the arguments used to prove the lemma of Riesz and simple trigonometric identities.

From (37) we readily see that

$$
\begin{aligned}
1-\left|L_{0}\left(A_{k}^{*} \mathbf{t}\right)\right|^{2}-\left|L_{1}\left(A_{k}^{*} \mathbf{t}\right)\right|^{2}= & 1-\cos ^{8}\left((2 m-1) \pi t_{1}\right) \\
& -\sin ^{8}\left((2 m-1) \pi t_{1}\right) .
\end{aligned}
$$

We define

$$
\begin{aligned}
q(t):= & 1-\cos ^{8}((2 m-1) \pi t) \\
& -\sin ^{8}((2 m-1) \pi t), \quad t \in \mathbb{R} .
\end{aligned}
$$

If $s:=\cos ((2 m-1) \pi t)$, we see that $q(t)=D(\cos ((2 m-1) \pi t))$, where $D(s)=-2 s^{8}+4 s^{6}-6 s^{4}+4 s^{2}$. Since $4-6 y+4 y^{2}-2 y^{3}=$ $-2(y-1)(y-c)(y-\bar{c})$, where $c=(1+i \sqrt{7}) / 2$, we conclude that

$$
D(s)=-2 s^{2}\left(s^{2}-1\right)\left(s^{2}-c\right)\left(s^{2}-\bar{c}\right) .
$$

Setting $z=e^{i(2 m-1) \pi t}$ and bearing in mind that $|z|=1$, this yields

$$
\begin{aligned}
q(t)= & -2\left[\left(\frac{z+z^{-1}}{2}\right)^{2}\right]\left[\left(\frac{z+z^{-1}}{2}\right)^{2}-1\right] \\
& \times\left[\left(\frac{z+z^{-1}}{2}\right)^{2}-c\right]\left[\left(\frac{z+z^{-1}}{2}\right)^{2}-\bar{c}\right] \\
= & -2(2 z)^{-8}\left(z^{2}+1\right)^{2}\left[\left(z^{2}+1\right)^{2}-4 z^{2}\right] B(z) \\
= & -2(2 z)^{-8}\left(z^{4}-1\right)^{2} B(z),
\end{aligned}
$$

where

$$
B(z)=\left[\left(z^{2}+1\right)^{2}-4 c z^{2}\right]\left[(z+1)^{2}-4 \bar{c} z^{2}\right] .
$$

Let

$$
z_{0}=\frac{1}{2}(2 \sqrt{7}+4 \sqrt{2})^{1 / 2}(1+i)
$$

Then $z_{0}$ is a zero of $\left(z^{2}+1\right)^{2}-4 c z^{2}$ and $\overline{z_{0}}$ is a zero of $(z+$ $1)^{2}-4 \bar{c} z^{2}$.

From the discussion in [17] or [14] we see that if $z=e^{i \pi t}$, then

$$
B(z)=\frac{1}{\left|z_{0}\right|^{2}}\left|\left(z-z_{0}\right)\left(z-\overline{z_{0}}\right)\left(z-\frac{1}{z_{0}}\right)\left(z-\frac{1}{\overline{z_{0}}}\right)\right|^{2} .
$$

Since $q(t) \geq 0$, we have

$$
q(t)=|q(t)|=2^{-7}\left|z^{4}-1\right|^{2} B(z) .
$$


Thus, a choice for $L\left(A_{k}^{*} \mathbf{t}\right)$ is

$$
\begin{aligned}
L\left(A_{k}^{*} \mathbf{t}\right):= & \frac{1}{8 \sqrt{2}\left|z_{0}\right|}\left(e^{i 4(2 m-1) \pi t_{1}}-1\right) \\
& \times\left(e^{i(2 m-1) \pi t_{1}}-z_{0}\right)\left(e^{i(2 m-1) \pi t_{1}}-\overline{z_{0}}\right) \\
& \times\left(e^{i(2 m-1) \pi t_{1}}-\frac{1}{z_{0}}\right)\left(e^{i(2 m-1) \pi t_{1}}-\frac{1}{\overline{z_{0}}}\right) .
\end{aligned}
$$

We now set

$$
\begin{aligned}
Q_{1}(\mathbf{t})=\frac{1}{\sqrt{2}}[ & 1-\cos ^{8}\left((2 m-1) \pi t_{1}\right) \\
& \left.\quad \cos ^{4}\left((2 m-1) \pi t_{1}\right) \sin ^{4}\left((2 m-1) \pi t_{1}\right)\right],
\end{aligned}
$$

$$
\begin{aligned}
Q_{2}(\mathbf{t})=\frac{e^{i 2 \pi t_{1}}}{\sqrt{2}}[1 & -\cos ^{8}\left((2 m-1) \pi t_{1}\right) \\
& \left.+\cos ^{4}\left((2 m-1) \pi t_{1}\right) \sin ^{4}\left((2 m-1) \pi t_{1}\right)\right],
\end{aligned}
$$

$$
Q_{3}(\mathbf{t})=-\frac{1}{2} \cos ^{4}\left((2 m-1) \pi t_{1}\right) \overline{L\left(A_{k}^{*} \mathbf{t}\right)}
$$

where $L\left(A_{k}^{*} \mathbf{t}\right)$ is defined by (52) with $z_{0}$ given by (49). If $\widehat{\phi}$ is defined by (24) with $n=2$,

$$
\widehat{\psi_{\ell}}\left(A_{k}^{*} \mathbf{t}\right):=Q_{\ell}(\mathbf{t}) \widehat{\phi}(\mathbf{t}), \quad \ell=1,2,3
$$

and $\Psi=\left\{\psi_{1}, \psi_{2}, \psi_{3}\right\}$ is the set of inverse Fourier transforms of the functions $\widehat{\psi}_{\ell}$ defined in the preceding displayed identity, then $\Psi$ is a tight framelet in $L^{2}\left(\mathbb{R}^{2}\right)$ with dilation factor $A_{k}$, and the functions $\psi_{\ell}$ have compact support.

\section{Acknowledgment}

A. San Antolín was partially supported by no. PB94-0153. R. A. Zalik is grateful to Instituto Argentino de Matemática for its hospitality during the completion of this work.

\section{References}

[1] K. Gröchenig and W. R. Madych, "Multiresolution analysis, Haar bases, and self-similar tilings of $R^{n}$," IEEE Transactions on Information Theory, vol. 38, no. 2, part 2, pp. 556-568, 1992.

[2] B. Han, "On dual wavelet tight frames", Applied and Computational Harmonic Analysis, vol. 4, no. 4, pp. 380-413, 1997.

[3] A. Ron and Z. Shen, "Affine systems in $L_{2}\left(\mathbb{R}^{d}\right)$ : the analysis of the analysis operator," Journal of Functional Analysis, vol. 148, no. 2, pp. 408-447, 1997.

[4] A. Ron and Z. Shen, "Compactly supported tight affine spline frames in $L_{2}\left(\mathbb{R}^{d}\right)$," Mathematics of Computation, vol. 67, no. 221, pp. 191-207, 1998.

[5] A. Ron and Z. W. Shen, "Construction of compactly supported afine frames in $L_{2}\left(\mathbb{R}^{d}\right)$," in Advances in Wavelets, K. S. Lau, Ed., pp. 27-49, Springer, New York, NY, USA, 1998.
[6] K. Gröchenig and A. Ron, "Tight compactly supported wavelet frames of arbitrarily high smoothness," Proceedings of the American Mathematical Society, vol. 126, no. 4, pp. 1101-1107, 1998.

[7] B. Han, "Compactly supported tight wavelet frames and orthonormal wavelets of exponential decay with a general dilation matrix," Journal of Computational and Applied Mathematics, vol. 155, no. 1, pp. 43-67, 2003.

[8] A. San Antolin and R. A. Zalik, "Some smooth compactly supported tight framelets," Communications on Pure and Applied Mathematics, vol. 3, no. 3, pp. 345-353, 2012.

[9] C. K. Chui, W. He, and J. Stöckler, "Compactly supported tight and sibling frames with maximum vanishing moments," Applied and Computational Harmonic Analysis, vol. 13, no. 3, pp. 224262, 2002 .

[10] I. Daubechies, B. Han, A. Ron, and Z. Shen, "Framelets: MRAbased constructions of wavelet frames," Applied and Computational Harmonic Analysis, vol. 14, no. 1, pp. 1-46, 2003.

[11] B. Han, "Nonhomogeneous wavelet systems in high dimensions," Applied and Computational Harmonic Analysis, vol. 32, no. 2, pp. 169-196, 2012.

[12] B. Han, "Pairs of frequency-based nonhomogeneous dual wavelet frames in the distribution space," Applied and Computational Harmonic Analysis, vol. 29, no. 3, pp. 330-353, 2010.

[13] M.-J. Lai and J. Stöckler, "Construction of multivariate compactly supported tight wavelet frames," Applied and Computational Harmonic Analysis, vol. 21, no. 3, pp. 324-348, 2006.

[14] P. Wojtaszczyk, A Mathematical Introduction to Wavelets, vol. 37 of London Mathematical Society Student Texts, Cambridge University Press, Cambridge, UK, 1997.

[15] R. S. Strichartz, "Wavelets and self-affine tilings," Constructive Approximation, vol. 9, no. 2-3, pp. 327-346, 1993.

[16] J. C. Lagarias and Y. Wang, "Haar type orthonormal wavelet bases in $R^{2}$," The Journal of Fourier Analysis and Applications, vol. 2, no. 1, pp. 1-14, 1995.

[17] I. Daubechies, Ten Lectures on Wavelets, vol. 61, Society for Industrial and Applied Mathematics, Philadelphia, Pa, USA, 1992.

[18] T. N. T. Goodman, C. A. Micchelli, G. Rodriguez, and S. Seatzu, "Spectral factorization of Laurent polynomials," Advances in Computational Mathematics, vol. 7, no. 4, pp. 429-454, 1997. 


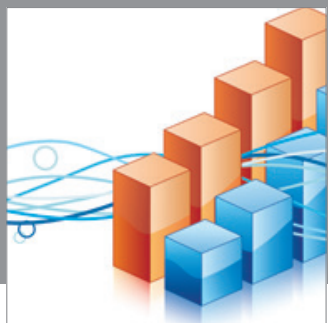

Advances in

Operations Research

mansans

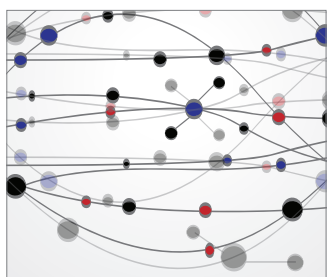

The Scientific World Journal
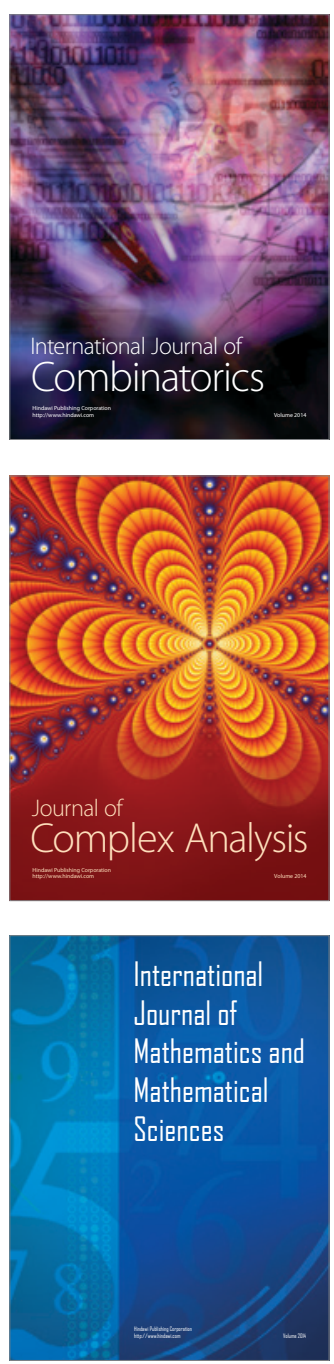
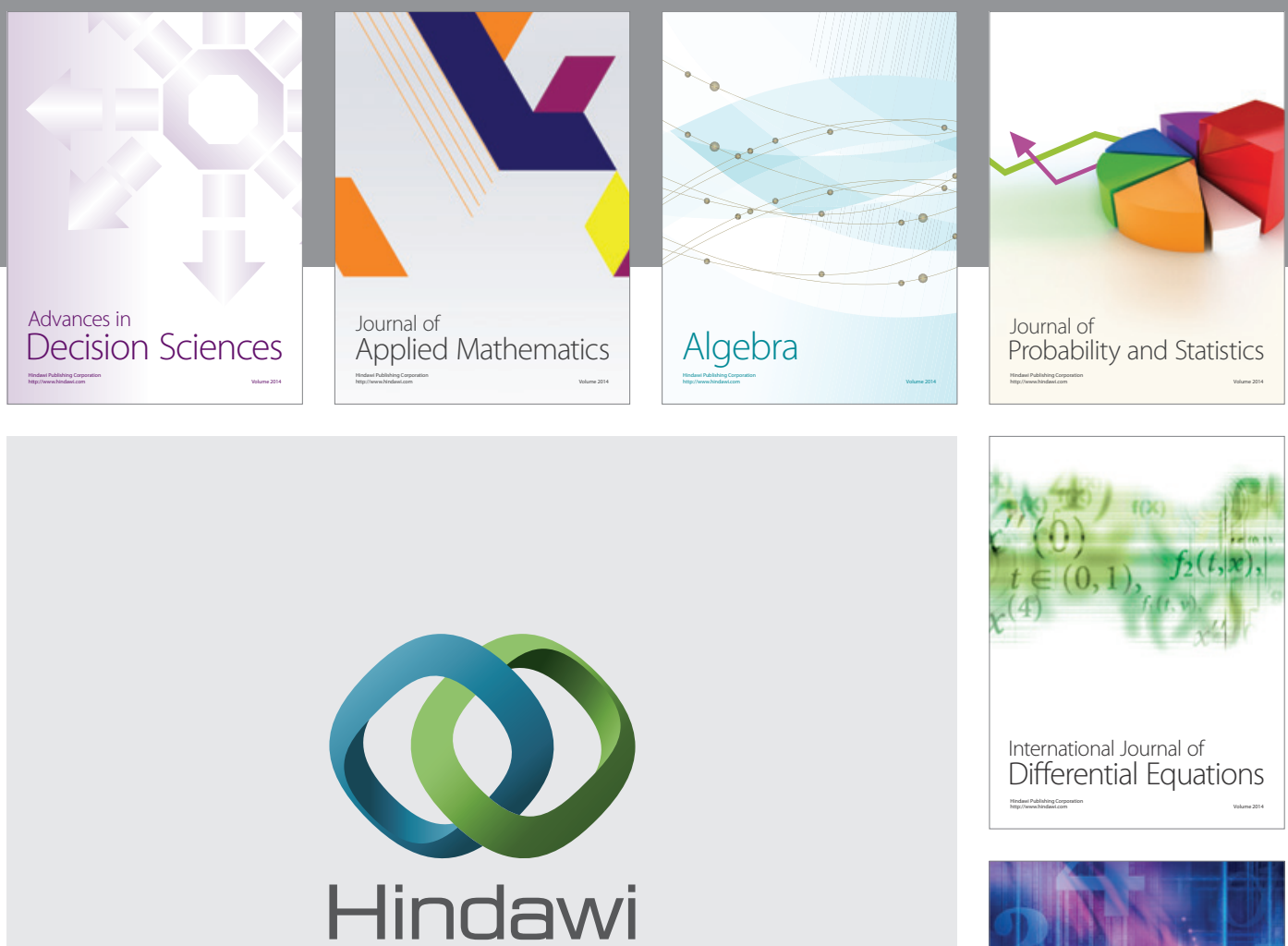

Submit your manuscripts at http://www.hindawi.com
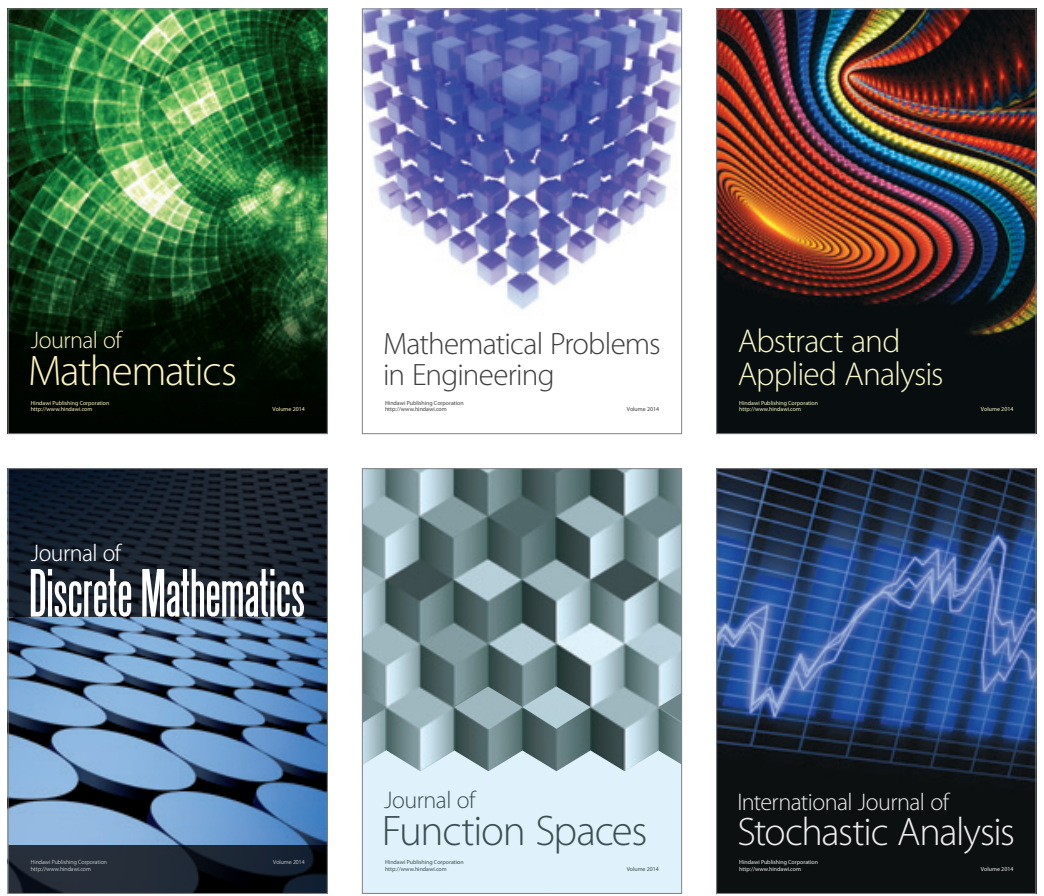

Journal of

Function Spaces

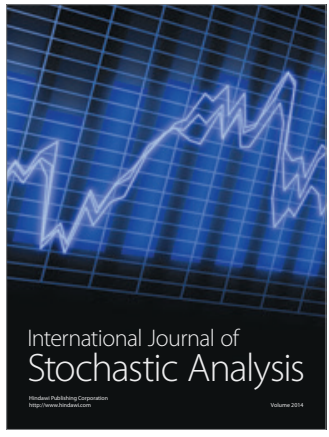

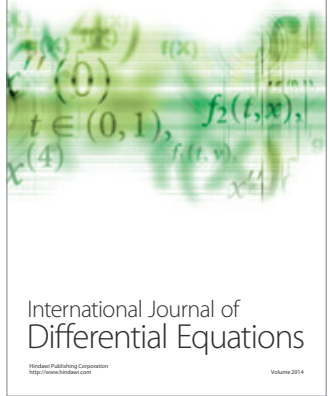
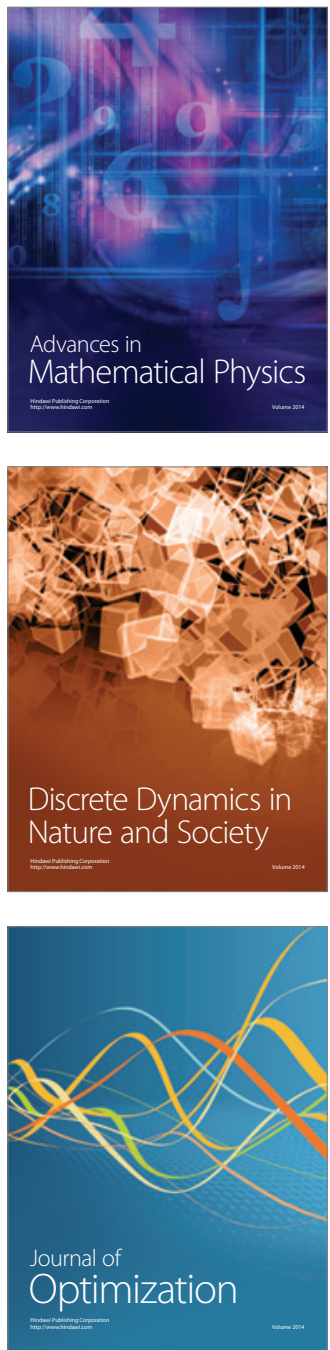